\title{
Refining bimodal microstructure of materials with MSTRUCT
}

\author{
Z. Matěj, ${ }^{1, a)}$ A. Kadlecová, ${ }^{1}$ M. Janeček, ${ }^{2}$ L. Matějová, ${ }^{3,4}$ M. Dopita, ${ }^{5}$ and R. Kužel ${ }^{1}$ \\ ${ }^{I}$ Department of Condensed Matter Physics, Faculty of Mathematics and Physics, Charles University in Prague, Ke Karlovu 5 , \\ 12116 Praha 2, Czech Republic \\ ${ }^{2}$ Department of Physics of Materials, Faculty of Mathematics and Physics, Charles University in Prague, Ke Karlovu 5, \\ 12116 Praha 2, Czech Republic \\ ${ }^{3}$ Institute of Environmental Technology, VŠB-Technical University of Ostrava, 17. listopadu 15/2172, 70833 Ostrava, \\ Czech Republic \\ ${ }^{4}$ Department of Catalysis and Reaction Engineering, Institute of Chemical Process Fundamentals of the ASCR, v.v.i., \\ Rozvojová 135, 16502 Praha 6, Czech Republic \\ ${ }^{5}$ Institute of Materials Science, TU Bergakademie Freiberg, Gustav-Zeuner-Strasse 5, 09599 Freiberg, Germany
}

(Received 2 June 2014; accepted 8 September 2014)

\begin{abstract}
The possibilities of modelling the diffraction profiles from bimodal microstructure in computer program MSTRUCT are demonstrated on two examples. A special "Double Component" profile effect can be utilized for such problems. At first it was applied to an analysis of a mixture of two nanocrystalline anatase powders with different crystallite sizes and the relative ratio of both components was determined from X-ray diffraction data. In the second case study, diffraction peaks from a pure polycrystalline copper sample treated by equal channel angular pressing were fitted using a two-phase model of large recrystallized defect-free grains and ultrafine crystallites with high dislocation density. The method is shown to be suitable for determination of the relative fraction of the microstructural components as well as other parameters (e.g. dislocation density). (c) 2014 International Centre for Diffraction Data. [doi:10.1017/S0885715614000852]
\end{abstract}

Key words: XRD, bimodal, crystallite size, dislocations, recrystallization

\section{INTRODUCTION}

The bimodal microstructure is quite common for many types of polycrystalline materials. Simple metals, particularly pure copper (Gubicza et al., 2005; Balogh et al., 2006), when processed by strong deformation, consists of ultrafine grained crystallites accompanied by large defect-free grains. This is related to thermal stability of these materials, recovery, and recrystallization processes (Kužel et al., 2013). In fact, it is not a speciality of copper and metallic materials (Singh et al., 2013; Zha et al., 2013; Dirras et al., 2014). Plasmadeposited microcrystalline silicon films (Ram et al., 2009), sintered $\mathrm{ZnO}$ (Kojdecki et al., 2007), or $\mathrm{BaTiO}_{3}$ nanocrystals produced by hydrothermal synthesis (Zhu et al., 2005) can possess a structure of crystallites of clearly different sizes and shapes. Typical diffraction profiles from such materials are narrow close to the peak centre, but they have broad tails (Gubicza et al., 2005; Kužel et al., 2013). In extreme cases even inflection points are visible. It can be difficult to approximate such peaks by standard profile functions, often super-Lorentzians are necessary. However, the best approach is to respect their nature and include some types of bimodal structure into the physical model itself. Crystallographic software dedicated specifically to the line profile analysis (LPA) (Leoni et al., 2006; Lutterotti et al., 2007; Coelho et al., 2011) offer several options, whereas in typical Rietveld computer programs (Rodriguez-Carvajal and Roisnel, 2004), the

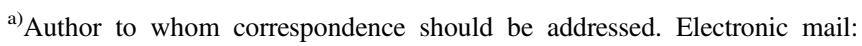
matej@karlov.mff.cuni.cz
}

most preferred choice is to generate two crystalline phases with same atomic structure but different line-broadening models, where the correlations between parameters are resolved by setting constraints on them. As the MSTRUCT (Matěj and Kužel, 2009) belongs rather to the first group. The use of a specific broadening effect for the bimodal microstructure description is demonstrated here on a model case of the sample with anatase $\left(\mathrm{TiO}_{2}\right)$ crystallites with two different sizes (Matěj et al., 2011). On the contrary, in some cases the increased degrees of freedom of the second approach using two phases with different microstructure and constrained parameters - is favourable. This is illustrated here on the refinement of the powder diffraction data from recrystallized deformed copper (Kužel et al., 2013). The use of parameters constraints in MSTRUCT has not been published yet. Therefore it is also briefly described here together with a mathematical background behind it.

\section{MSTRUCT - PHYSICAL EFFECTS}

MSTRUCT (Matěj and Kužel, 2009, which can be downloaded from http://www.xray.cz/mstruct) is a typical Rietveld (Rietveld, 1969) program implementing the whole profile modelling method (WPPM) (Ribárik et al., 2001, 2004; Scardi and Leoni, 2002) and dedicated also for powder diffraction analysis of thin films. It is a derivative/extension of the FOX/ObjCryst project (Favre-Nicolin and Černý, 2002) and at present it implements several models for diffraction line-broadening, shift, and intensity corrections (Table I). Some of the effects: residual stress and refraction correction 
TABLE I. List of physical effects implemented in MSTRUCT.

\begin{tabular}{|c|c|c|c|}
\hline Type & Effect name & Short description & Reference \\
\hline \multirow[t]{4}{*}{ Common physical } & Instrumental & $\begin{array}{l}\text { Instrumental broadening described by asymmetric } \\
\text { pseudoVoigt function and Cagliotti polynomial }\end{array}$ & Matěj et al., 2010 \\
\hline & $\begin{array}{l}\text { PseudoVoigt(A) } \\
\quad \text { (microstrain) }\end{array}$ & $\begin{array}{l}\text { Phenomenological model using for microstrain broadening } \\
\text { the pseudoVoigt function defined in real (A) or reciprocal } \\
\text { space }\end{array}$ & Matěj et al., 2010 \\
\hline & Anisotropic microstrain ${ }^{\mathrm{a}}$ & Same as pseudoVoigtA with $h k l$ anisotropy from Popa (1998) & $\begin{array}{l}\text { Popa 1998; Valeš } \\
\text { et al., } 2014\end{array}$ \\
\hline & Size log-normal & $\begin{array}{l}\text { Line broadening from spherical crystallites with log-normal } \\
\text { distribution of their diameters }\end{array}$ & $\begin{array}{l}\text { Ribárik et al., 2001; } \\
\text { Scardi and Leoni, } \\
2002\end{array}$ \\
\hline \multirow{4}{*}{$\begin{array}{l}\text { Thin films, stress, } \\
\text { texture }\end{array}$} & Absorption correction & Thin film absorption correction (without Fresnel correction). & Matěj et al., 2010 \\
\hline & Residual stress (XECs) & $\begin{array}{l}\text { Isotropic bi-axial simple stress model for thin films with } \\
\text { X-ray elastic constants according to Popa (2000) }\end{array}$ & $\begin{array}{l}\text { Popa, 2000; Matěj } \\
\quad \text { et al., } 2010\end{array}$ \\
\hline & Refraction correction & Line position correction for refraction on film interface & Matěj et al., 2010 \\
\hline & Texture calculator & $\begin{array}{l}\text { Numerical texture calculator for ODF projection; } \\
\text { multi-component texture model }\end{array}$ & Matěj et al., 2008 \\
\hline \multirow[t]{4}{*}{ Special physical } & Dislocations & $\begin{array}{l}\text { Wilkens model of restrictedly random dislocation } \\
\text { distribution and dislocation broadening anisotropy (cubic } \\
\text { and } h c p \text { systems) }\end{array}$ & $\begin{array}{l}\text { Ribárik et al., 2001; } \\
\text { Scardi and Leoni, } \\
2002\end{array}$ \\
\hline & Stacking faults & $\begin{array}{l}\text { Warren model of profiles from stacking faults in } f c c \text { and } h c p \\
\text { crystals }\end{array}$ & $\begin{array}{l}\text { Velterop et al., } 2000 \\
\text { Scardi and Leoni, } \\
2002\end{array}$ \\
\hline & Sticks/rods/platelets ${ }^{\mathrm{a}}$ & Size broadening from rod-like crystallites with elliptic basis & $\begin{array}{l}\text { Brunátová et al., } \\
\text { submitted }\end{array}$ \\
\hline & Histogram distribution & Histogram-like crystallite size distribution & Matěj et al., 2011 \\
\hline \multirow[t]{3}{*}{ Auxiliary } & Arbitrary texture & $\begin{array}{l}\text { Refinable intensities of individual } h \mathrm{kl} \text { peaks not constrained } \\
\text { by structure model }\end{array}$ & Lutterotti et al., 2007 \\
\hline & $H K L$ pseudoVoigt & $\begin{array}{l}\text { Refinable shift and additional broadening of individual } h k l \\
\text { peaks }\end{array}$ & $\begin{array}{l}\text { To be published, } \\
\text { Matěj, } 2011\end{array}$ \\
\hline & Double component ${ }^{\mathrm{a}}$ & $\begin{array}{l}\text { General double-component profile for modelling bimodal } \\
\text { microstructure (bimodal size distribution, profiles after } \\
\text { recrystallization, etc.) }\end{array}$ & Described here \\
\hline
\end{tabular}

${ }^{\mathrm{a}}$ Effects implemented in 2012-2014.

for thin films were described in Matěj et al. (2010). In particular, the last effect in Table I is designed for description of bimodal microstructure. However, let us at first briefly describe the technical details concerning the parameters constraints in MSTRUCT.

\section{MSTRUCT - LEAST-SQUARE CONSTRAINTS}

The core of the original FOX/ObjCryst computer program (Favre-Nicolin and Černý, 2002) is a parallel tempering global optimization algorithm for structure solution. For the Rietveld refinement, it is more suitable to use the standard least-square (LSQ) method. In fact, this algorithm is already a part of FOX/ ObjCryst and it is also a standard optimization procedure in MSTRUCT (Matěj et al., 2010). In addition, it was further extended to include constraints between the LSQ parameters here.

In each step of the LSQ algorithm, one is looking for an improvement $\delta_{a}$ of a current solution a by solving a set of equations

$$
\alpha \cdot \delta_{\mathrm{a}}=\beta
$$

where $\alpha$ is the $n \times n$ Hessian matrix, $\beta$ is the right-hand side of the LSQ problem and $n$ is the number of parameters. The singular value decomposition (SVD) method is used in
MSTRUCT for finding the inverse of $\alpha$. We would like to set some linear relations within the set a of the refined parameters now. Let $C$ be a matrix of such constraints. The first constraint for a change $\delta_{\mathrm{a}}$ of the model parameters can be written as

$$
C_{11} \delta_{\mathrm{a} 1}+\cdots+C_{1 i} \delta_{\mathrm{a} i}+\cdots=0
$$

and instead of Eq. (1) an extended problem must be solved

$$
\left(\begin{array}{l}
\alpha \\
C
\end{array}\right) \cdot \delta_{\mathrm{a}}=\left(\begin{array}{l}
\beta \\
0
\end{array}\right)
$$

where the matrix on the left-hand side is not square, but still the inverse of the problem can be calculated using, e.g. the generalized SVD algorithm of van Loan (1976). In the next step, the refined parameter limits are checked, i.e. if the new solution $\left(a+\delta_{\mathrm{a}}\right)$ is outside of the allowed limits, the solution is truncated to fit within them. The generalized SVD method is used here again to construct an appropriate projection to parameters subspace fulfilling Eq. (2).

In Figure 1, a part of the MSTRUCT input parameters file is depicted. LSQ constraints are specified by the code word @ LSQConstraint followed by a colon, an arbitrary title for the particular constraint and a number of parameters related to it. Names of the related parameters and coefficients of matrix $C$ are specified on the next lines. Figure 1 illustrates 


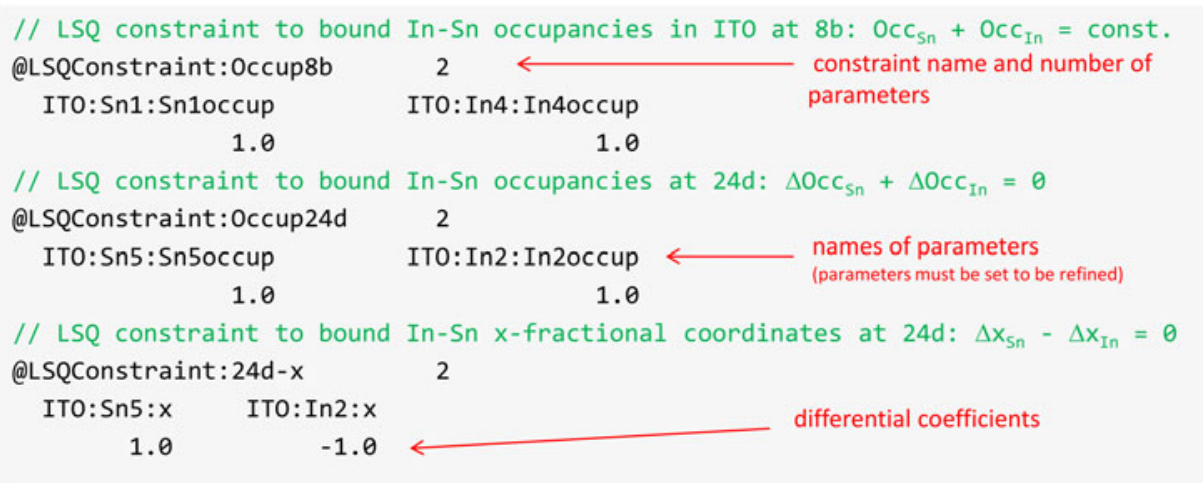

Figure 1. (Colour online) Example of an input parameter section with definition of LSQ-constraints for occupancies and atom coordinates in crystal structure of tin-doped indium oxide.

an example of constraints applied to occupancies (Occ) and positions $(x)$ of $(\mathrm{Sn}, \mathrm{In})$ atoms in the lattice of tin-doped indium oxide (ITO)

$$
\begin{gathered}
\operatorname{Occ}_{\mathrm{Sn}}+\operatorname{Occ}_{\mathrm{In}}=\text { const. } \\
\Delta x_{\mathrm{Sn}}-\Delta x_{\mathrm{In}}=0
\end{gathered}
$$

\section{DOUBLE-COMPONENT PROFILE}

The tool designed especially for description of the bimodal microstructure is the Double-component profile effect. The appropriate code word is DoubleCompReflProf. Usually, individual effects are convoluted together in MSTRUCT. Within DoubleCompReflProf two effects can be summed together with an appropriate weight factor (w), which is the only model parameter. Diffraction profiles $I(s)$ in reciprocal space units $s=2 \sin (\theta) / \lambda-2 \sin \left(\theta_{h k l}\right) / \lambda$ and Fourier coefficients $A(x)$ can then be written as

$$
\begin{gathered}
I(s)=(1-w) \cdot I_{1}(s)+w \cdot I_{2}(s) \\
A(x)=(1-w) \cdot A_{1}(x)+w \cdot A_{2}(x)
\end{gathered}
$$

where the integrals over $I_{i}(s)$ are normalized to one and the Fourier coefficients $A(x)$ are at zero real-space variable $x=0$ normalized as $A(x=0)=1$. This implies $w$ is the volume fraction of the second microstructure component.

An application of the DoubleCompReflProf effect is illustrated here on the analysis of the artificial powder sample the mixture of anatase crystallites of two different sizes $\left(\left\langle D_{1}\right\rangle=6.7 \mathrm{~nm}\right.$ and $\left.\left\langle D_{2}\right\rangle=22.6 \mathrm{~nm}\right)$. The sample as well as the synthesis conditions were described in Matěj et al. (2011, 2013). DoubleCompReflProf is a "virtual" effect. It induces no shift or broadening of simulated diffraction lines itself. It is used as a parent object of children profiles, which can already represent real broadening effects. Such a hierarchical structure is schematically depicted in Figure 2. The topmost parent, DoubleCompReflProf, has two virtual children ReflProf objects and their children are real broadening effects. The crystallites are assumed to be spherical and their bimodal size is represented by two independent log-normal distributions. The both anatase microstructure phases share the same strain broadening effect to reduce the number of model parameters. The appropriate section of the input parameters file is depicted in Figure 3, the fitted powder pattern in Figure 4 and the resulting crystallite size distribution in Figure 5.

With the bimodal size model, the goodness of fit quality $\mathrm{GoF}=1.35$ was achieved for the studied sample. The results of other approaches are summarized in Table II. The bimodal model gives almost as good solution as the refinement of the general size distribution histogram (Matěj et al., 2011) and significantly better fit than a standard model, assuming monodisperse particles. The agreement between determined size distribution parameters $(M, S)$ with reference samples is quite good and even excellent when recalculated to areaand volume-averaged dimensions $\left(\langle D\rangle_{\mathrm{A}}\right.$ and $\left.\langle D\rangle_{\mathrm{V}}\right)$. On the contrary, the model of monodisperse particles gives wrong values of the distribution parameters. Area- and volumeweighted crystallite sizes are quite similar in all the cases, showing that they are not volatile to the choice of a particular method. Also the volume fractions of small and large crystallites refined from the bimodal model and the histogram

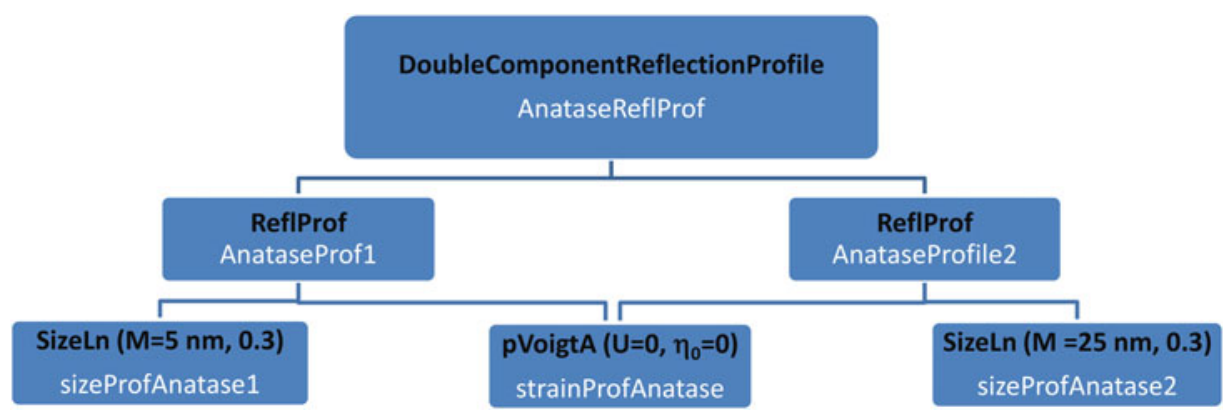

Figure 2. (Colour online) Schematic diagram of broadening models hierarchy for description of the bimodal microstructure using DoubleCompReflProf effect. 
// (total number - including virtual and unused effects)

$/ / 1$ (parent prof) +2 (child profs) +3 ( 2 size and 1 strain comps. $)=6$

// the 1st phase - Parent Reflection Profile Object - double component broadening effect

DoubleCompReflProf AnataseReflProf

AnataseReflProf1

AnataseReflProf2

0.5

1 broadening component type

name of the first component

name of the second component

fraction of the second component

top parent effect (1-yes,0-no)
Figure 3. (Colour online) Preview of an input parameter section with definition of the bimodal broadening effect for anatase phase - implementation of a part of diagram from Figure 2.

// the 1st phase - Child Reflection Profile Object 1 - Size + Strain broadenning

ReflProf AnataseReflProf1

2

sizeProfAnatase 1

strainProfAnatase

0 broadening component type

number of additional broadening effects

broadening component name

broadening component name

top parent effect (1-yes,0-no) fitting (Matěj et al., 2011) are very similar. The both values are 5 vol.\% smaller than the nominal value. Other aspects of the two-component microstructure, which were not considered here, can be a reason for such discrepancy. Furthermore, Figure 5 illustrates a well-known fact that the diffraction is a volume-sensitive method. The arithmetic fraction of large crystallites is so small ( $f \approx 2-5 \%$, Table II), that these crystallites are hardly visible in the main axes of
Figure 5(a), but the volume fraction of these crystallites is about 60\%; see Figure 5(b).

DoubleCompReflProf has advantages in that it naturally follows the idea of bimodal microstructure, is adding only a single-model parameter and still it is quite general. However, it is just the nature why DoubleCompReflProf effect is not as useful as the authors intended. In many cases, the bimodal crystallite size distribution is related to other differences in

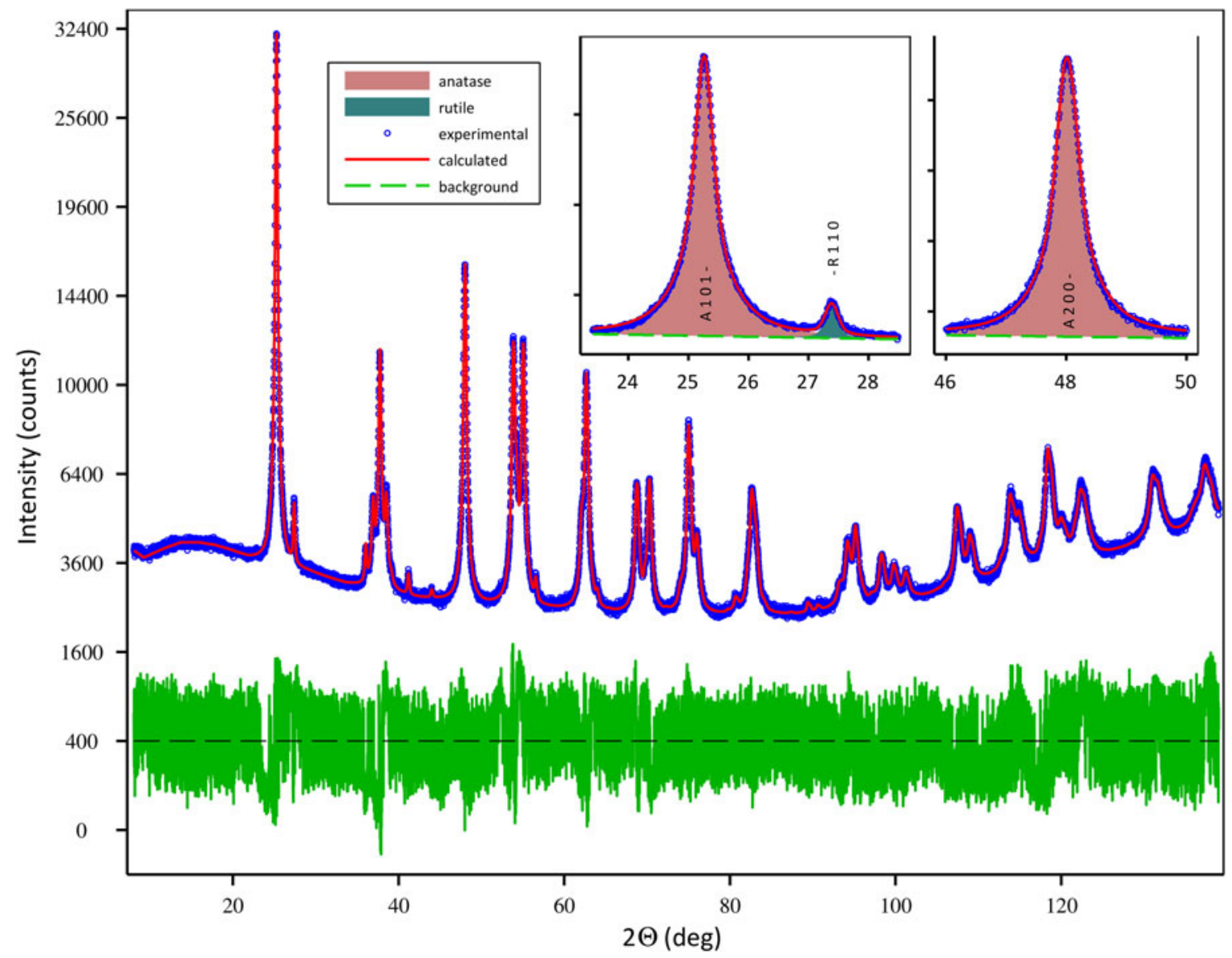

Figure 4. (Colour online) Whole powder pattern modelling fit of $\mathrm{TiO}_{2}$ powder sample with the bimodal size distribution. 


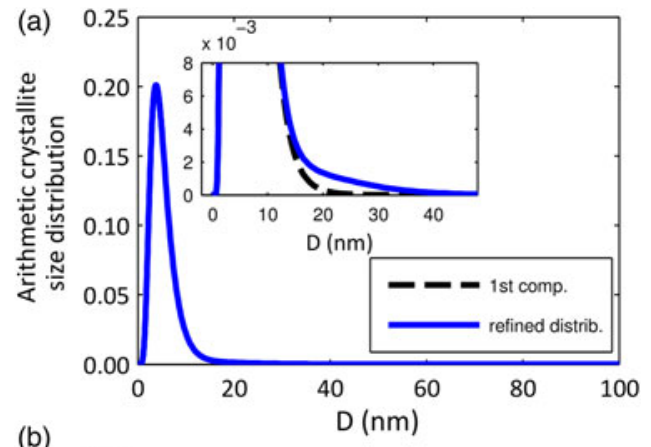

(b)

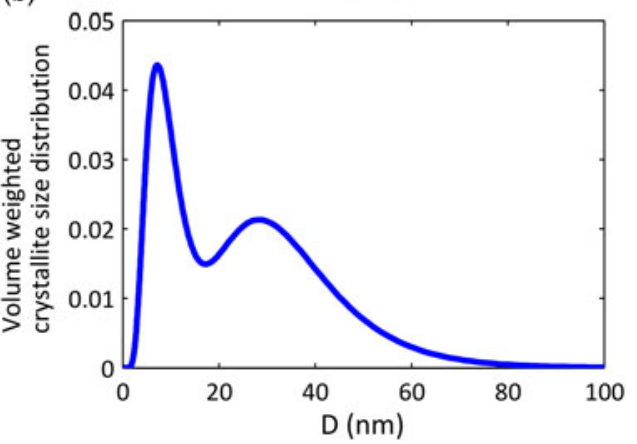

Figure 5. (Colour online) (a) Arithmetic and (b) volume weighted crystallite size distribution as refined for sample in Figure 4.

micro(structure) of two crystallite components. Crystallites of different size can have distinct lattice parameters (Matěj et al., 2013) or possess different preferred orientation. Then it is worthwhile to reconsider if the model of two independent crystalline phases with the same crystal structure, but different microstructure, could propose more possibilities to better describe the experimental data.

\section{TWO-PHASE MODEL - BIMODAL MICTROSTRUCTURE OF COPPER}

In Kužel et al. (2013; idem, to be published) a thermal stability of pure copper and copper with a small addition of $\mathrm{Zr}$ processed by equal channel angular pressing (ECAP) was studied in situ by the XRD. Samples after processing by severe deformation show strongly broadened diffraction peaks, mainly owing to the presence of dislocations (density

TABLE II. Comparison of results from MSTRUCT fitting of bimodal nanocrystalline $\mathrm{TiO}_{2}$ powder. (GoF ... goodness of fit; $M_{i}, S_{i} \ldots$ size distribution parameters - mean crystallites size $M \exp \left(S^{2} / 2\right) ; w, f \ldots$ volume and arithmetic fractions of the second microstructure phase; $\langle D\rangle_{\mathrm{A}},\langle D\rangle_{\mathrm{V}} \ldots$ area and volume weighted mean crystallite sizes).

\begin{tabular}{lllll}
\hline \hline & Bimodal & $\begin{array}{c}\text { Histogram } \\
(20 \text { bins })\end{array}$ & Monodisperse & Reference \\
\hline $\mathrm{GoF}$ & 1.35 & 1.31 & 1.41 & - \\
$M_{1}(\mathrm{~nm})$ & $4.7(2)$ & - & $2.75(6)$ & $6.2(1)$ \\
$S_{1}$ & $0.46(2)$ & - & $0.814(4)$ & $0.384(3)$ \\
$M_{2}(\mathrm{~nm})$ & $22(1)$ & - & - & $20.7(3)$ \\
$S_{2}$ & $0.37(1)$ & - & - & $0.398(5)$ \\
$w(\mathrm{vol} \%)$ & $60(2)$ & $61(5)$ & 0 & $66(1)$ \\
$f(\%)$ & 2 & - & 0 & 5 \\
$\langle D\rangle_{\mathrm{A}}(\mathrm{nm})$ & 14.4 & - & 14.4 & 16.8 \\
$\langle D\rangle_{\mathrm{V}}(\mathrm{nm})$ & 25.2 & - & 28.0 & 27.3 \\
\hline \hline
\end{tabular}

TABLE III. Comparison of goodness of fit factors (GoF) for different models for the $\mathrm{Cu}$ sample annealed for $40 \mathrm{~h}$ at $125^{\circ} \mathrm{C}$.

\begin{tabular}{lccc}
\hline \hline & \multicolumn{2}{c}{ Independent for the recrystallized phase } & \\
\cline { 2 - 3 } Model type & Line shift & Texture & GoF \\
\hline Uniform & \multicolumn{2}{c}{ Average structure } & 4.6 \\
Bimodal & Not & Not & 3.4 \\
Bimodal & Yes & Not & 3.15 \\
Two phase & Not & Yes & 1.90 \\
Two phase & Yes & Yes & 1.89 \\
\hline \hline
\end{tabular}

$\left.10^{15} \mathrm{~m}^{-2}\right)$. Minor effects are stacking faults $(\leq 1 \%)$ and crystallites size (80-200 nm). Pure $\mathrm{Cu}$ after annealing for approximately $1 \mathrm{~h}$ at relatively low temperature $\left(125^{\circ} \mathrm{C}\right)$ and also $\mathrm{Cu}+\mathrm{Zr}$ (annealed at $380^{\circ} \mathrm{C}$ ) show strongly the bimodal structure of diffraction peaks as described already, e.g. by Gubicza et al. (2005). At a fixed temperature, the strongly broadened peaks from the deformed structure continuously transform with time into the narrow peaks (Kužel et al., 2013, idem, to be published). The ultrafine grain structure created by strong deformation process is volatile to thermal annealing. Dislocations tend to annihilate in grain boundaries, while small crystallites "recrystallize" into large almost defect-free grains. Narrow diffraction line profiles close to the peaks maxima come from this recrystallized fraction and can be well described by the instrumental function, with no need of additional broadening effects. During modelling in fact for both these components peak line positions corrections are also applied to get the best fit to experimental data. For this purpose, the so-called HKLpseudoVoigt effect (Table I) is used. With this effect additional pseudo-Voigt function can be convoluted on the selected $h k l$ reflection producing more broadening, or in the case here, only a shift of the $h k l$ lines. These shifts are negligible in comparison with diffraction line width. They were reported already in Matěj et al. (2009), were confirmed by X-ray diffraction (XRD) measurements in the parallel beam geometry (Matěj, 2011) and their origin is most likely related to so-called "intergranular strains" (Popa, 2008). Up to this point the DoubleCompReflProf can be used to describe all these effects. However, the ECAP materials show often a strong texture (Kužel et al., 2010), which is difficult to describe. The recrystallized grains orientation does not need to follow exactly the orientation distribution of crystallites just after the deformation process and changes slightly during the in situ annealing. It was already mentioned this is a weak point of the double-component profile effect.

Table III summarizes the $G o F$ factors for different models, which were used to fit diffraction profiles from a pure $\mathrm{Cu}$ ECAP sample annealed in situ at $125^{\circ} \mathrm{C}$ for $40 \mathrm{~h}$. An example fit is depicted in Figure 6 . The standard model assuming uniform microstructure gives a very poor fit to experimental data. Including the bimodal microstructure model was an improvement, which was highly enhanced by dropping the assumption the deformed and recrystallized components have same texture. The DoubleCompReflProf effect cannot be used, as it is too much restrictive.

In some cases, the "two-phase" model must be preferred. For its generality, it is necessary to pay with some user inconvenience, e.g. when maintaining the input parameters files. 


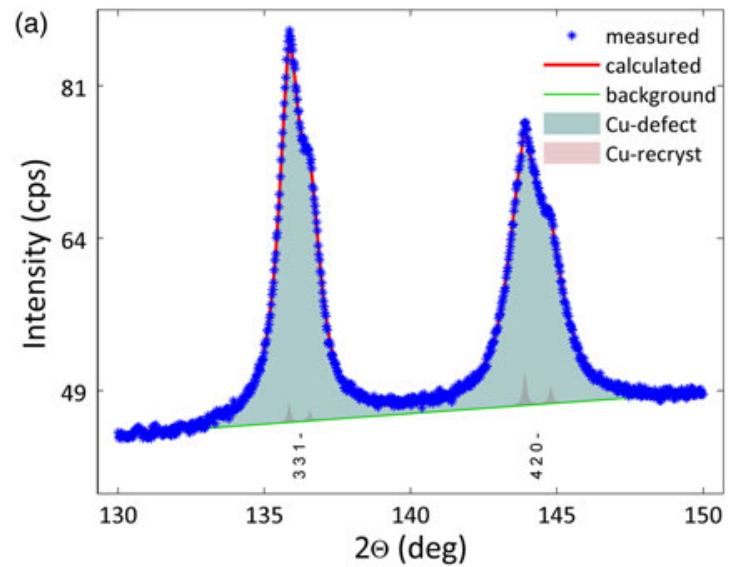

(b)

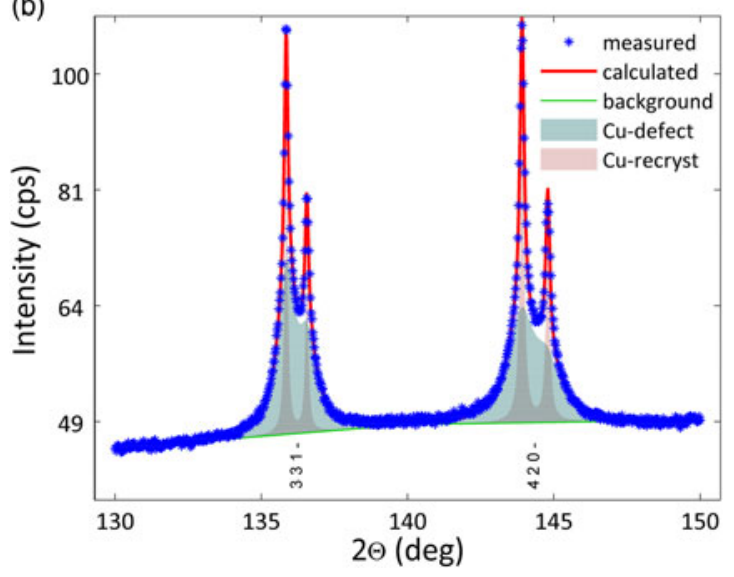

Figure 6. (Colour online) Fit of high-angle reflections of pure $\mathrm{Cu}$ sample (a) as processed by ECAP and (b) after annealing for $40 \mathrm{~h}$ at $125^{\circ} \mathrm{C}$. Sample microstructure was described by an almost equal volume fraction of recrystallized defect-free grains and ultra-fine crystallites with high density of defects in (b) whereas the recrystallized fraction is very small in (a).

\section{CONCLUSION}

Two approaches of modelling bimodal microstructure in MSTRUCT were presented. The first one, which is based on the Double-component profile effect, was used to simulate bimodal crystallite size distribution in the $\mathrm{TiO}_{2}$ powder. Its advantages are the natural way that it represents the bimodal structure and simplicity of its usage. For some applications, it can be limiting and it can be preferable to rather introduce two-crystal phases with the similar atomic structure, but distinct microstructure. This was the case of copper sample deformed by ECAP, which after annealing shows bimodal microstructure consisting of large defect-free grains and ultrafine crystallites that have a high density of dislocations. The generality of the second approach used for modelling of the copper sample is at the expense of some user convenience as it implies incorporation of constraints and an increase of complexity in setting the input model. With both these methods, it was possible to determine not only the fractions of individual components, but also their other parameters, as mean crystallite size or dislocation density.

\section{ACKNOWLEDGEMENTS}

This work was supported by the Grant Agency of the Czech Republic (Project no. 14-23274S) and within the Charles University Research Center "Physics of Condensed
Matter and Functional Materials" (no. UNCE 204023/2012). The authors are also very grateful to Dr. Petr Lukáš from Nuclear Physics Institute of the ASCR, v.v.i. for discussion concerning the origin of line shifts in ECAP samples.

Balogh, L., Gubicza, J., Hellmig, R. J., Estrin, Y., and Ungar, T. (2006). "Thermal stability of the microstructure of severely deformed copper," Z. Kristallogr. Suppl. 23, 381-386.

Brunátová, T., Matěj, Z., Oleynikov, P., Veselý, J., Daniš, S., Popelková, D., and Kužel, R. (submitted). "Thermal stability of titanate nanorods and titania nanowires formed from titanate nanotubes by heating," Mater. Charact.

Coelho, A. A., Evans, J., Evans, I., Kern, A., and Parsons, S. (2011). "The TOPAS symbolic computation system," Powder Diffr. 26, S22-S25.

Dirras, G., Tingaud, D., Csiszar, G., Gubicza, J., Couque, H., and Mompiou, F. (2014). "Characterization of bulk bimodal polycrystalline nickel deformed by direct impact loadings," Mater. Sci. Eng. A, Struct. Mater. Prop. Microstruct. Process. 601, 48-57.

Favre-Nicolin, V. and Černý, R. (2002). "FOX, 'free objects for crystallography': a modular approach to ab initio structure determination from powder diffraction,” J. Appl. Crystallogr. 35, 734-743.

Gubicza, J., Balogh, L., Hellmig, R. J., Estrin, Y., and Ungar, T. (2005). "Dislocation structure and crystallite size in severely deformed copper by X-ray peak profile analysis," Mater. Sci. Eng. A, Struct. Mater. Prop. Microstruct. Process. 400, 334-338.

Kojdecki, M. A., Mielcarek, W., Prociow, K., and Warycha, J. (2007). "Crystalline microstructure of zinc oxide powder sintered into varistor body," Solid State Phenom. 130, 237-240.

Kužel, R., Janeček, M., Matěj, Z., Čížek, J., Dopita, M., and Srba, O. (2010). "Microstructure of equal-channel angular pressed $\mathrm{Cu}$ and $\mathrm{Cu}-\mathrm{Zr}$ samples studied by different methods," Metall. Mater. Trans. A, Phys. Metall. Mater. Sci. 41, 1174-1190.

Kužel, R., Matěj, Z. and Janeček, M. (2013). "In-situ X-Ray diffraction study of thermal stability of $\mathrm{Cu}$ and $\mathrm{Cu}-\mathrm{Zr}$ samples processed by ECAP," Mater. Sci. Forum 753, 279-284.

Kužel, R., Kadlecová, A., Matěj, Z., Janeček, M., Stráská, J., and Dopita, M. (in press). "Time and temperature stability of $\mathrm{Cu}$ and $\mathrm{Cu}-\mathrm{Zr}$ samples processed by ECAP studied by in-situ X-ray diffraction."

Leoni, M., Confente, T. and Scardi, P. (2006). "PM2 K: a flexible program implementing Whole Powder Pattern Modelling,” Z. Kristallogr. Suppl. 23, 249-254.

Lutterotti, L., Bortolotti, M., Ischia, G., Lonardelli, I. and Wenk, H.-R. (2007). "Rietveld texture analysis from diffraction images," Z. Kristallogr. Suppl. 26, 125-130.

Matěj, Z. (2011). Structure of submicrocrystalline materials studied by X-ray diffraction. PhD thesis, Faculty of Mathematics and Physics, Charles University in Prague.

Matěj, Z. and Kužel, R. (2009). MStruct-program/library for MicroStructure analysis by powder diffraction. http://www.xray.cz/mstruct/ (May 31, 2014).

Matěj, Z., Nichtová, L. and Kužel, R. (2008). "Expanding FOX for microstructure analysis," Mater. Struct. Chem. Biol. Phys. Technol. 15, 46-49.

Matěj, Z., Kužel, R., Dopita, M., Janeček, M., Čížek, J. and Brunátová, T. (2009). "XRD profile analysis of ECAP $\mathrm{Cu}$ and $\mathrm{Cu}+\mathrm{Zr}$ samples," Int. J. Mater. Res. 100, 880-883.

Matěj, Z., Kužel, R. and Nichtová, L. (2010). "XRD total pattern fitting applied to study of microstructure of $\mathrm{TiO}_{2}$ films," Powder Diffr. 25, 125-131.

Matěj, Z., Matějová, L., Novotný, F., Drahokoupil, J. and Kužel, R. (2011). "Determination of crystallite size distribution histogram in nanocrystalline anatase powders by XRD," Z. Kristallogr. Proc. 1, 87-92.

Matěj, Z., Matějová, L. and Kužel, R. (2013). "XRD analysis of nanocrystalline anatase powders prepared by various chemical routes: correlations between micro-structure and crystal structure parameters," Powder Diffr. 28 (S2), S161-S183.

Popa, N. C. (1998). "The (hkl) dependence of diffraction-line broadening caused by strain and size for all Laue groups in Rietveld refinement," J. Appl. Crystallogr. 31, 176-180. 
Popa, N. C. (2000). "Diffraction-line shift caused by residual stress in polycrystal for all Laue groups in classical approximations," J. Appl. Crystallogr. 33, 103-107.

Popa, N. C. (2008). "Microstructural properties: texture and macrostress effects," in Powder Diffraction: Theory and Practice, edited by R. E. Dinnebier and S. J. L. Billinge (RCS Publishing, Cambridge), pp. 332-375.

Ram, S. K., Islam, Md N., Kumar, S. and Roca i Cabarrocas, P. (2009). "Evidence of bimodal crystallite size distribution in mu c-Si:H films," Mater. Sci. Eng. B, Adv. Funct. Solid-State Mater. 159-160, 34-37.

Ribárik, G., Gubicza, J. and Ungár, T. (2004). "Correlation between strength and microstructure of ball-milled Al-Mg alloys determined by X-ray diffraction," Mater. Sci. Eng. A 387-389, 343-347.

Ribárik, G., Ungár, T. and Gubicza, J. (2001). "MWP-fit: a program for multiple whole-profile fitting of diffraction peak profiles by ab initio theoretical functions," J. Appl. Crystallogr. 34, 669-676.

Rietveld, H. M. (1969). "A profile refinement method for nuclear and magnetic structures," J. Appl. Crystallogr. 2, 65-71.

Rodriguez-Carvajal, J. and Roisnel, T. (2004). "Line broadening analysis using FullProf*: determination of microstructural properties," Mater. Sci. Forum 443-444, 123-126.
Scardi, P. and Leoni, M. (2002). "Whole powder pattern modelling," Acta Crystallogr. A 58, 190-200.

Singh, A., Katakam, S., Ilavsky, J., Dahotre, N. B. and Harimkar, S. P. (2013). "Nanocrystallization in spark plasma sintered $\mathrm{Fe}_{48} \mathrm{Cr}_{15} \mathrm{Mo}_{14} \mathrm{Y}_{2} \mathrm{C}_{15} \mathrm{~B}_{6}$ bulk amorphous alloy," J. Appl. Phys. 114, 054903.

Valeš, V., Matějová, L., Matěj, Z., Brunátová, T. and Holý, V. (2014). "Crystallization kinetics study of cerium titanate $\mathrm{CeTi}_{2} \mathrm{O}_{6}$," J. Phys. Chem. Solids 75, 265-270.

van Loan, Ch. F. (1976). "Generalizing the singular value decomposition," SIAM J. Numer. Anal. 13, 76-83.

Velterop, L., Delhez, R., de Keijser, Th. H., Mittemeijer, E. J. and Reefman, D. (2000). "X-ray diffraction analysis of stacking and twin faults in f.c.c. metals: a revision and allowance for texture and non-uniform fault probabilities," J. Appl. Crystallogr. 33, 296-306.

Zha, M., Li, Y., Mathiesen, R. H., Bjorge, R. and Roven, H. J. (2013). "Annealing response of binary Al-7Mg alloy deformed by equal channel angular pressing," Mater. Sci. Eng. A, Struct. Mater. Prop. Microstruct. Process. 586, 374-381.

Zhu, X. H., Zhu, J. M., Zhou, S. H., Liu, Z. G., Ming, N. B. and Hesse, D. (2005). " $\mathrm{BaTiO}_{3}$ nanocrystals: Hydrothermal synthesis and structural characterization," J. Crystallogr. Growth 283, 553-562. 